\title{
ASYMPTOTIC BEHAVIOR OF EIGENVALUES AND EIGENFUNCTIONS OF STURM-LIOUVILLE PROBLEMS WITH COUPLED BOUNDARY CONDITIONS AND TRANSMISSION CONDITIONS
}

\author{
DAn Mu, Jiong Sun And Ji-Jun Ao
}

\begin{abstract}
The Sturm-Liouville (S-L) problems with coupled boundary conditions and transmission conditions are investigated. By defining a new Hilbert space which is related to the transmission conditions, the self-adjointness of the S-L problems in this associated Hilbert space is proved, and the asymptotic behavior of eigenvalues and eigenfunctions of the problem are described. We also give the condition for $\lambda$ being the eigenvalue of the S-L problems with coupled boundary conditions.
\end{abstract}

Mathematics subject classification (2010): 34L20, 47E05.

Keywords and phrases: Sturm-Liouville problems, eigenvalues, eigenfunctions, asymptotic formulas.

\section{REFERENCES}

[1] R. A. Adams And J. J. F. Fournier, Sobolev Spaces, Volume 140, Second Edition (Pure and Applied Mathematics), 2003.

[2] N. Altinisik, M. Kadakal, O. Sh. Mukhtarov, Eigenvalues and eigenfunctions of discontinuous Sturm-Liouville problems with eigenparameter-dependent boundary conditions, Acta Mathematica Hungarica, 102 (2004), 159-193.

[3] N. Altinisik, O. Sh. Mukhtarov, M. Kadakal, Asymptotic formulas for eigenfunctions of the Sturm-Liouville problems with eigenvalue parameter in the boundary conditions, Kuwait J. Sci. 39 (1A) (2012), 1-17.

[4] R. KH. Amirov, Eigenvalues and normalized eigenfunctions of discontinuous Sturm-Liouville problem with transmission conditions, J. Math. Anal. Appl., 317 (1) (2006), 163-176.

[5] K. Aydemir, O. Sh. Mukhtarov, Green's function method for self-adjoint realization of boundary-value problems with interior singularities, Abstract and Applied Analysis, (2013), Article ID 503267, 7 pages.

[6] K. AYDEMIR, Boundary value problems with eigenvalue-dependent boundary and transmission conditions, Boundary value problems, 1 (2014), 131.

[7] E. Bairamov, E. Ugurlu, The determinants of dissipative Sturm-Liouville operators with transmission conditions, Mathematical and Computer Modelling, 53 (2011), 805-813.

[8] M. Demirci, Z. AKdoĞAN, O. S. MuKhtarov, Asymptotic behavior of eigenvalues and eigenfunctions of one discontinuous boundary-value problem, International Journal of Computational Cognition, 2 (3) 2004, 101-113.

[9] W. N. EVERITT, D. RACE, On necessary and sufficient conditions for the existence of Caratheodory solutions of ordinary differential equations, Quaest. Math., 3 (1976), 507-512.

[10] W. N. EveritT, A. ZetTL, Sturm-Liouville differential operators in direct sum spaces, Rocky Mountain J. Math., 16 (3) (1986), 497-516.

[11] O. Sh. Mukhtarov, M. Kadakal and F. S. Muhtarov, Eigenvalues and normalized eigenfunctions of discontinuous Sturm-Liouville problem with transmission conditions, Reports on Mathematical Physics, 54 (1) (2004), 41-56.

[12] C. SHIEH, V. A. YURKO, Inverse nodal and inverse spectral problems for discontinuous boundary value problems, J. Math. Anal. Appl., 347 (2008), 266-272. 
[13] E. C. Titchmarsh, The Theory of Functions (2nd ed.), Oxford University Press, 1939.

[14] E. C. TITChMARSH, Eigenfunction Expansions Associated with Second-order Differential Equations, Clarendon Press, Oxford (1946).

[15] A. WAng, J. Sun, X. HaO, And S. Yao, Completeness of eigenfunctions of Sturm-Liouville problems with transmission conditions, Methods Appl. Anal., 16 (3) (2009), 299-312.

[16] C. YANG, Inverse nodal problems of discontinuous Sturm-Liouville operator, J. Differential Equations, 254 (2013), 1992-2014.

[17] V. A. YURKO, Boundary value problems with discontinuity conditions in an interior point of the interval, Diff. Equa., 36 (8) (2000), 1266-1269.

[18] A. ZettL, Sturm-Liouville Theory, AMS, Mathematical Surveys and Monographs vol. 121 (2005). 